\title{
VGLUT2 Is a Determinant of Dopamine Neuron Resilience in a Rotenone Model of Dopamine Neurodegeneration
}

\author{
${ }^{D}$ Silas A. Buck, ${ }^{1,2 *}$ Briana R. De Miranda, ${ }^{3,4 *}{ }^{\circledR}$ Ryan W. Logan, ${ }^{5,6}$ Kenneth N. Fish, ${ }^{2}$ J. Timothy Greenamyre, ${ }^{3,7}$ \\ and ${ }^{\circledR}$ Zachary Freyberg 2,8 \\ ${ }^{1}$ Center for Neuroscience, University of Pittsburgh, Pittsburgh, Pennsylvania, $15213,{ }^{2}$ Department of Psychiatry, University of Pittsburgh, Pittsburgh, \\ Pennsylvania, $15213,{ }^{3}$ Department of Neurology, University of Pittsburgh, Pittsburgh, Pennsylvania, $15260,{ }^{4}$ Department of Neurology, University of \\ Alabama at Birmingham, Birmingham, Alabama, 35294, ${ }^{5}$ Department of Pharmacology and Experimental Therapeutics, Boston University School of \\ Medicine, Boston, Massachusetts, 02118, ${ }^{6}$ Center for Systems Neurogenetics of Addiction, The Jackson Laboratory, Bar Harbor, Maine, 04609, \\ ${ }^{7}$ Pittsburgh Institute for Neurodegenerative Diseases, University of Pittsburgh, Pittsburgh, Pennsylvania, 15260, and ${ }^{8}$ Department of Cell Biology, \\ University of Pittsburgh, Pittsburgh, Pennsylvania, 15213
}

Parkinson's disease (PD) is characterized by progressive dopamine (DA) neuron loss in the SNc. In contrast, DA neurons in the VTA are relatively protected from neurodegeneration, but the underlying mechanisms for this resilience remain poorly understood. Recent work suggests that expression of the vesicular glutamate transporter 2 (VGLUT2) selectively impacts midbrain DA neuron vulnerability. We investigated whether altered DA neuron VGLUT2 expression determines neuronal resilience in rats exposed to rotenone, a mitochondrial complex I inhibitor and toxicant model of PD. We discovered that VTA/SNc DA neurons that expressed VGLUT2 are more resilient to rotenone-induced DA neurodegeneration. Surprisingly, the density of neurons with detectable VGLUT2 expression in the VTA and SNc increases in response to rotenone. Furthermore, dopaminergic terminals within the NAc, where the majority of VGLUT2-expressing DA neurons project, exhibit greater resilience compared with DA terminals in the caudate/putamen. More broadly, VGLUT2-expressing terminals are protected throughout the striatum from rotenone-induced degeneration. Together, our data demonstrate that a distinct subpopulation of VGLUT2-expressing DA neurons are relatively protected from rotenone neurotoxicity. Rotenone-induced upregulation of the glutamatergic machinery in VTA and SNc neurons and their projections may be part of a broader neuroprotective mechanism. These findings offer a putative new target for neuronal resilience that can be manipulated to prevent toxicant-induced DA neurodegeneration in PD.

Key words: dopamine; glutamate; neurodegeneration; rotenone; tyrosine hydroxylase; VGLUT2

Significance Statement

Environmental exposures to pesticides contribute significantly to pathologic processes that culminate in Parkinson's disease (PD). The pesticide rotenone has been used to generate a PD model that replicates key features of the illness, including dopamine neurodegeneration. To date, longstanding questions remain: are there dopamine neuron subpopulations resilient to rotenone; and if so, what are the molecular determinants of this resilience? Here we show that the subpopulation of midbrain dopaminergic neurons that express the vesicular glutamate transporter 2 (VGLUT2) are more resilient to rotenone-induced neurodegeneration. Rotenone also upregulates VGLUT2 more broadly in the midbrain, suggesting that VGLUT2 expression generally confers increased resilience to rotenone. VGLUT2 may therefore be a new target for boosting neuronal resilience to prevent toxicant-induced DA neurodegeneration in PD. 


\section{Introduction}

Parkinson's disease (PD) is a neurodegenerative movement disorder characterized by a loss of dopaminergic (DA) neurons in the SNc (Nussbaum and Ellis, 2003; de Lau and Breteler, 2006; Wood-Kaczmar et al., 2006). DA neurons of the VTA, however, are relatively protected from DA neurodegeneration (Surmeier, 2018). An important clue to resilience of VTA DA neurons is that a significant fraction of these cells express the vesicular glutamate transporter 2 (VGLUT2), which packages glutamate into synaptic vesicles (Shen et al., 2018; Mingote et al., 2019). In contrast, in the adult SNc, VGLUT2 ${ }^{+}$DA neurons constitute $<5 \%$ of the total DA neuron pool (Dal Bo et al., 2008; Bérubé-Carrière et al., 2009). Although the precise purpose and mechanisms of DA/glutamate corelease from these VGLUT2-expressing DA neurons remain controversial, $\mathrm{VGLUT}^{+}{ }^{+} \mathrm{DA}$ neurons are more likely to survive neurotoxic insults in several preclinical PD models, suggesting that VGLUT2 expression in DA neurons confers increased resilience (Dal Bo et al., 2008; Bérubé-Carrière et al., 2009; Shen et al., 2018; Steinkellner et al., 2018). Consistent with this, conditional VGLUT2 KO from DA neurons increases vulnerability to cell loss in response to DA neurotoxins (Shen et al., 2018; Steinkellner et al., 2018). Moreover, striatal VGLUT2 expression is upregulated both in preclinical models and clinical PD (Kashani et al., 2007; Dal Bo et al., 2008; Zheng et al., 2019). In all, these data suggest that DA neuron VGLUT2 expression modifies vulnerability.

While DA neurotoxin models of PD, including 6-OHDA and MPTP, have been carefully examined in studies of VGLUT2's roles in DA neuron vulnerability (Shen et al., 2018; Steinkellner et al., 2018), it remains unknown whether VGLUT2 is similarly implicated in vulnerability to rotenone, a pesticide and prototypical mitochondrial complex I inhibitor (Betarbet et al., 2000; Cannon et al., 2009). Unlike other toxicant models, rotenone causes significant and progressive deficits in the autophagy-lysosomal pathway, neuroinflammation, and accumulation of intracellular protein (e.g., $\alpha$-synuclein), recapitulating hallmark pathology observed in human PD (De Miranda et al., 2019; Rocha et al., 2020). Critically, environmental toxicants, such as rotenone, play important roles in causing sporadic PD and/or amplifying preexisting PD risk (Betarbet et al., 2000; Tanner et al., 2014; Kolber and Krüger, 2019). Indeed, environmental exposures to rotenone years before clinical symptoms may trigger the biological processes that lead to DA neurodegeneration (Tanner et al., 2014). The rotenone model can therefore reveal broader, shared mechanisms of action by which DA neuron VGLUT2 expression modifies vulnerability compared with stressors such as 6-OHDA which produce a narrower constellation of pathologic changes in dopaminergic cells, as well as offer new insights into environmental precipitants of sporadic PD (Betarbet et al., 2002; Cannon et al., 2009).

Here, using a combination of multiplex RNAscope and immunohistochemical approaches, we discovered that midbrain DA neurons that express VGLUT2 are more resilient to neurodegeneration caused by rotenone. In addition, rotenone treatment significantly increased the density of VGLUT2-immunoreactive puncta (i.e., putative glutamatergic terminals) in the VTA and SNc. Moreover, striatal nerve terminals expressing VGLUT2 are more resilient to rotenone-induced degeneration. Together, our findings suggest that VGLUT2 expression is a critical component of a more global survival response that offers protection from rotenone-induced neurodegeneration.

\section{Materials and Methods}

Animal husbandry

Adult 10-month-old male Lewis rats (Envigo) were separated into single-housing 2 weeks before the beginning of experiments and handled daily to minimize stress. This study was limited to male Lewis rats since the dosage of rotenone that causes severe, symptomatic DA neurodegeneration in male rats $(2.8 \mathrm{mg} / \mathrm{kg})$ does not cause dopaminergic degeneration in females (De Miranda et al., 2019), making it difficult to directly compare males versus females using the same rotenone dose. The rats were maintained under standard temperature-controlled conditions with a $12 \mathrm{~h}$ light-dark cycle, and food and water were available ad libitum. All animals were cared for in accordance with all appropriate animal care guidelines according to the National Institutes of Health Animal Care and Use Program as well as the Animal Research: Reporting of In Vivo Experiments guidelines for reporting animal research (Drummond et al., 2010). Moreover, every effort was made to ameliorate animal suffering. All experiments were approved by the University of Pittsburgh Institutional Animal Care and Use Committee.

\section{Rotenone administration}

Rotenone was dissolved in 2\% DMSO and Miglyol 812 (IOI Oleo) to reach a final concentration of $2.8 \mathrm{mg} / \mathrm{kg}$. Rotenone and vehicle $(2 \%$ DMSO and Miglyol 812) groups were randomly assigned and administered a daily intraperitoneal injection. Rotenone-treated animals were determined to have reached a motor behavioral endpoint once severe bradykinesia or a loss of $25 \%$ of body mass was observed. Once at endpoint, animals were killed with pentobarbital followed by decapitation. For immunohistochemistry experiments, animals were transcardially perfused with $4 \%$ PFA; and after $24 \mathrm{~h}$ fixation in PFA, brains were transferred to $30 \%$ sucrose and stored at $4^{\circ} \mathrm{C}$ until sectioned using a freezing microtome. For ISH experiments, brain tissue was flash frozen in powdered dry ice and stored at $-80^{\circ} \mathrm{C}$ before sectioning.

\section{Motor behavior}

Motor deficits were assessed daily using the Postural Instability Test (PIT), which can measure changes in forelimb motor dysfunction, including detection of the development of asymmetry (between left and right paws), as described previously (Woodlee et al., 2008; Cannon et al., 2009; De Miranda et al., 2018, 2019). Briefly, rats (vehicle: $N=3$; rotenone: $N=3$ ) were first habituated to handling for 2 weeks before the study to minimize stress. In performing the PIT, each rat was held with 1 forelimb immobilized, and the opposite forelimb was placed on a metered surface with texture to prevent slipping. The animal was slowly moved forward until the forelimb was in contact with the surface, forcing the subject to take a step (distance to trigger), and the distance (in centimeters) was recorded as the PIT. The PIT consisted of an average of three trials per day for each animal and was conducted until each rat reached its motor behavioral endpoint, defined as the rat's inability to successfully complete the PIT, by becoming akinetic. All behavioral tests were conducted by fully blinded investigators.

\section{Stereology}

Stereological analysis of dopamine neuron number in the SNc was performed by an experimenter blinded to the treatment group via a protocol adapted from Tapias et al. (Tapias et al., 2013; Tapias and Greenamyre, 2014) and further described previously (De Miranda et al., 2018, 2019; Zharikov et al., 2019; Rocha et al., 2020). Briefly, nigral tissue sections were stained for TH and counterstained with DAPI, since NeuN is not a reliable marker of DA neurons (Cannon and Greenamyre, 2009), and fluorescent Nissl NeuroTrace Dye (640; Thermo Fisher Scientific, Invitrogen). Sections were imaged on a Nikon 90i upright fluorescence microscope equipped with high NA plan fluor/apochromat objectives, Renishaw linear encoded microscope stage (Prior Electronics) and Q-imaging Retiga cooled CCD camera (Center for Biological Imaging, University of Pittsburgh). Collected images were then processed using Nikon NIS-Elements Advanced Research software (version 4.5, Nikon), which enabled unbiased, automated quantitation of colocalizing DAPI, TH, and Nissl-positive stains. 
Table 1. Primary antibody information

\begin{tabular}{lllll}
\hline Antigen & Antibody catalog information & Company & Host species & Immunohistochemistry/immunocytochemistry concentration \\
\hline TH & AB1542 & EMD Millipore & Sheep & $1: 2000$ \\
VGLUT2 & Ab79157 & Abcam & Mouse & $1: 500$ \\
VGLUT2 & 135403 & Synaptic Systems & Rabbit & $1: 250$ \\
$\beta$-Actin & Ab8226 & Abcam & Mouse & $1: 1000$ \\
\hline
\end{tabular}

\section{FISH}

Multiplex RNAscope mRNA detection. Our multiplex RNAscope detection of mRNA expression employed protocols and reagents established for fresh-frozen tissue by Advanced Cell Diagnostics (Newark, California) as described previously (Erben and Buonanno, 2019). Brains (vehicle: $N=5$; rotenone: $N=4$ ) were cryostat-sectioned, and two tissue sections $(20 \mu \mathrm{m})$ were collected at $-5.3 \mathrm{~mm}$ from bregma, followed by mounting onto Superfrost Plus slides (Thermo Fisher Scientific). Slides were immersed in chilled 4\% PFA and then switched to $50 \%$ ethanol, followed by $70 \%$ ethanol and then $100 \%$ ethanol. A hydrophobic barrier was drawn on the slide around the tissue, and then slides were incubated in Pretreatment 3 to cover the tissue. Probes for rat VGLUT2 (Channel 1, Alexa-488; ACD) and rat TH (Channel 2, Atto 550, ACD) mRNAs were added to completely cover the tissue, and slides were incubated at $40^{\circ} \mathrm{C}$ in an EZ Hybridization oven (ACD). After incubation, slides were washed twice and incubated with Amplification Reagent (Amp1) at $40^{\circ}$ C. The wash and incubation steps were repeated with Amp2, Amp3, and Amp4 reagents. Slides were then incubated with DAPI at room temperature for cell nucleus labeling and mounted with Prolong Gold Antifade Mountant (Thermo Fisher Scientific, Invitrogen).

Fluorescence microscopy and image analysis of mRNA expression. Images were acquired with an Olympus IX83 inverted fluorescence microscope (Olympus America) equipped with a Hamamatsu ORCAFlash4.0 CCD camera (Hamamatsu) and high-precision BioPrecision2 $\mathrm{XYZ}$ motorized stage with linear XYZ encoders (Ludl Electronic Products) using a $60 \times 1.4$ NA SC oil immersion objective. Image stacks $(2048 \times 2048$ pixels; $0.2 \mu \mathrm{m} z$ steps $)$ were taken over the total tissue thickness in the VTA and SNc and were randomly selected using a grid of $100 \mu \mathrm{m}^{2}$ frames spaced by $500 \mu \mathrm{m}$, leading to imaging of 8-18 sites per hemisphere. Image collection and processing were performed using Slidebook 6.0 (Intelligent Imaging Innovations) and MATLAB (The MathWorks) software. First, a Gaussian channel was constructed for each channel by calculating a difference of Gaussians using $\sigma$ values of 0.7 and 2. Images were then separated into quantitative TIFF files of each individual channel and transferred to the HALO image analysis platform equipped with a fluorescent ISH add-on (version 3.0, Indica Labs). Using HALO software, DAPI-stained nuclei were quantified as any $40-500 \mu \mathrm{m}^{2}$ object; VGLUT2 ${ }^{+}$and $\mathrm{TH}^{+}$puncta were quantified as any $0.1-0.5 \mu \mathrm{m}^{2}$ object. After determining the average puncta density levels for each group (VGLUT2/vehicle: 2.7 puncta/DAPI-stained nucleus; VGLUT2/rotenone: 3.0 puncta/DAPI-stained nucleus; TH/vehicle: 27.7 puncta/DAPI-stained nucleus; TH/rotenone: 6.0 puncta/DAPIstained nucleus), thresholds were set as follows: 10 mRNA grains for VGLUT2, 20 mRNA grains for TH. These thresholds represented $\sim 3$ times the smaller observed density level and were used as cutoffs for positive cells to eliminate false positives that occur because of nuclei neighboring positive cells. Therefore, a DAPI-stained nucleus was deemed VGLUT2 $^{+}$if it contained a minimum of 10 VGLUT2 puncta within $5 \mu \mathrm{m}$ of the nucleus edge, and a cell was determined to be $\mathrm{TH}^{+}$if it contained a minimum of $20 \mathrm{TH}$ puncta within $5 \mu \mathrm{m}$ of the nucleus edge.

\section{Immunohistochemistry}

Staining. Serial sections ( $35 \mu \mathrm{m}$ thickness, vehicle: $N=4-7$; rotenone: $N=5-8$ for SNc, vehicle: $N=3$; rotenone: $N=3$ for VTA, vehicle: $N=6$, rotenone: $N=3-6$ for striatum) encompassing the entire ventral midbrain and/or striatum in a 1/6 series were cut on a freezing microtome. Uniform anatomic landmarks between animals were identified based on bregma area. We selected sections that aligned with bregma $-5.30 \mathrm{~mm}$, encompassing the medial SN. All sections were maintained at $-20^{\circ} \mathrm{C}$ in cryoprotectant before performing immunohistochemistry. Free-floating brain tissue sections were washed with PBS, and then immunostained with TH and VGLUT2 antibodies (Table 1). The specificity of both TH and VGLUT2 antibodies has been previously described (Herzog et al., 2006; Zhang et al., 2015; Wang et al., 2016; Strand et al., 2018). Sections were incubated with anti-mouse 555, anti-rabbit 488, and/or anti-sheep 647 secondary antibodies raised in donkey (Invitrogen). Sections were then mounted onto glass slides for imaging. Additionally, we used a "primary antibody delete" (secondary antibody-only) stained section to subtract background fluorescence.

Confocal microscopy and image analysis of protein expression in the ventral midbrain. Images were acquired using an Olympus BX61 confocal microscope equipped with $60 \times$ and $100 \times$ oil immersion objectives; equipment was controlled by Fluoview 1000 software (Olympus America). Imaging parameters, including laser power and exposure times, were held constant across all samples, and quantitative fluorescence measurements were thoroughly monitored to ensure that images contained no saturated pixels. As noted above, the sections we selected and analyzed for TH and VGLUT2 staining all aligned with bregma $-5.30 \mathrm{~mm}$, encompassing the medial SN. Analysis was performed using Nikon NIS-Elements Advanced Research software (version 4.5) and ImageJ (National Institutes of Health). At least 6 images were analyzed per slice per animal, averaging 9-15 neurons per image $(\sim 180$ cells per animal, per histologic stain). After background subtraction, fluorescent objects were size gated between 0.05 and $0.7 \mu \mathrm{m}^{3}$ (Rocco et al., 2016). Notably, all puncta analyzed were fully contained within each virtual counting frame and thus unaffected by sectioning angle.

For distinguishing and quantifying the VGLUT2 ${ }^{+}$puncta localized to midbrain $\mathrm{TH}^{+}$cells versus on DA cell membranes (e.g., presynaptic inputs), an automated detection function of Nikon Elements Advanced Research Software (NIS-AR, version 4.5) was used to: (1) identify the ROI boundaries of $\mathrm{TH}^{+}$dopaminergic neurons using an autodetect setting to limit user bias, based on the presence of TH immunofluorescence in a $100 \times$ brain tissue image. (2) NIS-AR parameters were set to exclude any puncta touching the ROI boundary, as these puncta could not be ruled out as synaptic inputs from other neurons. (3) NIS-AR autodetected VGLUT2 ${ }^{+}$puncta within the ROI boundary of each $\mathrm{TH}^{+}$neuron, with defined parameters to exclude size ( $<10$ pixels). Results were reported as numbers of $\mathrm{TH}^{+}$cells, numbers of puncta within $\mathrm{TH}^{+}$cells (i.e., number of objects per $\mathrm{TH}^{+}$cell), and intensity of $\mathrm{TH}^{+}$and VGLUT2 ${ }^{+}$puncta.

\section{Confocal microscopy and image analysis of protein expression in the} striatum

For studies of striatal $\mathrm{TH}^{+}$and VGLUT2 ${ }^{+}$puncta colocalization, images were collected on an Olympus IX81 inverted microscope equipped with an Olympus spinning disk confocal unit, Hamamatsu ORCA-Flash4.0 CCD camera and high precision BioPrecision2 XYZ motorized stage with linear XYZ encoders using a $60 \times 1.40$ NA SC oil immersion objective. Equipment was controlled by SlideBook 6.0 software. 3D image stacks $(1024 \times 1024$ pixels, $0.25 \mu \mathrm{m} z$ steps $)$ were acquired over $50 \%$ of the total thickness of the tissue sections. Caudate/putamen $(\mathrm{CPu})$ and NAc were identified using previously described stereotactic coordinates (Paxinos and Watson, 2007), and image stacks were randomly selected using a sampling grid of $100 \mu \mathrm{m}^{2}$ frames spaced by $1250 \mu \mathrm{m}$, leading to imaging of 8-12 sites per section. Each fluorescent channel was deconvolved using Autoquant's Blind Deconvolution algorithm. Then, a Gaussian channel was made for each deconvolved channel by calculating a difference of Gaussians using $\sigma$ values of 0.7 and 2. The Gaussian channel was used for data segmentation only, which was performed via MATLAB. For segmentation, object masks were created using a size-gating of $0.05-0.7 \mu \mathrm{m}^{3}$ as performed previously (Rocco et al., 2016). Density 
of overlapping VGLUT2 ${ }^{+}$and $\mathrm{TH}^{+}$puncta (at least one voxel) was analyzed in the $\mathrm{CPu}$ and NAc. To confirm the levels of puncta overlap were not because of random chance from overlaying two densely labeled channels, TH- and VGLUT2-immunoreactive pixels were selected using Costes' automatic thresholding (Costes et al., 2004), grouped into blocks of $7 \times 7$ pixels, and randomized 200 times. The average correlation coefficient (Pearson's $r$ ) of the 200 randomized permutations was compared with the correlation coefficients of TH and VGLUT2 pixels in the actual image. For all immunohistochemical analyses, all fluorescent puncta analyzed were fully contained within the sampling grid.

\section{N27 cell rotenone treatment}

The N27-A rat dopaminergic neuronal cell line was plated onto glass coverslips coated with poly-D-lysine (Thermo Fisher Scientific) at a density of $1 \times 10^{6}$ cells per well and cultured using standard DMEM media plus L-glutamine (Invitrogen, Thermo Fisher Scientific) with $1 \times$ penicillin/streptomycin and 10× FBS supplements. Twenty-four hours after plating, N27-A cells were treated with $100 \mathrm{~nm}$ rotenone or vehicle (DMSO) for a $24 \mathrm{~h}$ period and subsequently fixed using $4 \%$ PFA. The cells were stained for $\beta$-actin (ab8226, Abcam) and VGLUT2 (135403, Synaptic Systems), followed by corresponding secondary antibodies AlexaFluor-488 and -555 (Thermo Fisher Scientific). Immunocytochemistry images were obtained using an Olympus BX61 confocal microscope and Fluoview 1000 software.

\section{Statistical analysis}

All data are expressed as mean \pm SEM. Postural instability, immunohistochemistry, and ISH data were analyzed using Kruskal-Wallis tests with Mann-Whitney post hoc comparisons. Dunn's correction for multiple comparisons was used where appropriate. Pixel correlations were compared with random distributions using a Wilcoxon matched-pairs signed-rank test. For all statistics, significance was defined as $p<0.05$. All statistical analyses were conducted using SPSS (version 25, IBM) and GraphPad Prism software (version 8.2, GraphPad Software).

\section{Results}

Rotenone treatment leads to severe motor deficits and loss of $\mathrm{TH}^{+}$DA neurons

Age-matched adult rats underwent a chronic administration of rotenone $(2.8 \mathrm{mg} / \mathrm{kg} / \mathrm{d}$, i.p.) until motor and/or behavioral endpoints were reached, as this method of rotenone administration has previously been shown to reproduce key motor and pathologic features of PD (De Miranda et al., 2018, 2019; Rocha et al., 2020). Consistent with our earlier studies, we found decreased Nissl-stained cells in the SNc of the rotenone-treated rats (Fig. $1 A)$, confirming that rotenone indeed caused marked midbrain DA neurodegeneration. This was accompanied by rotenoneinduced decreases in immunoreactivity to $\mathrm{TH}$, the rate-limiting enzyme for DA biosynthesis and canonical DA neuron marker, in both the dopaminergic cell bodies of the SNc and their striatal projections (Fig. $1 B$ ). In line with this, unbiased stereological quantification demonstrated a $38 \%$ loss of $\mathrm{TH}^{+} \mathrm{DA}$ neurons in the $\mathrm{SNc}$ compared with the vehicle control $(U=0, p=0.0025$; Fig. 1C). Moreover, TH fluorescence intensity significantly diminished among $\mathrm{TH}^{+}$nerve terminals projecting into the dorsolateral striatum in response to rotenone versus vehicle $(U=0, p=0.0022$; Fig. 1D). To further confirm midbrain DA neurodegeneration, we examined whether our rotenone regimen produced motor behavior deficits using the PIT, an assay sufficiently sensitive to detect subtle daily changes in motor behavior caused by progressive DA neuron loss (Woodlee et al., 2008; Cannon et al., 2009; De Miranda et al., 2018). We showed that progressively worsening performance on the PIT was correlated with duration of rotenone administration $\left(r^{2}=0.95, \mathrm{H}_{14}=41.8, p<0.001\right.$; Fig. $\left.1 E\right)$. Dunn's post hoc comparisons revealed that rotenone-treated rats had
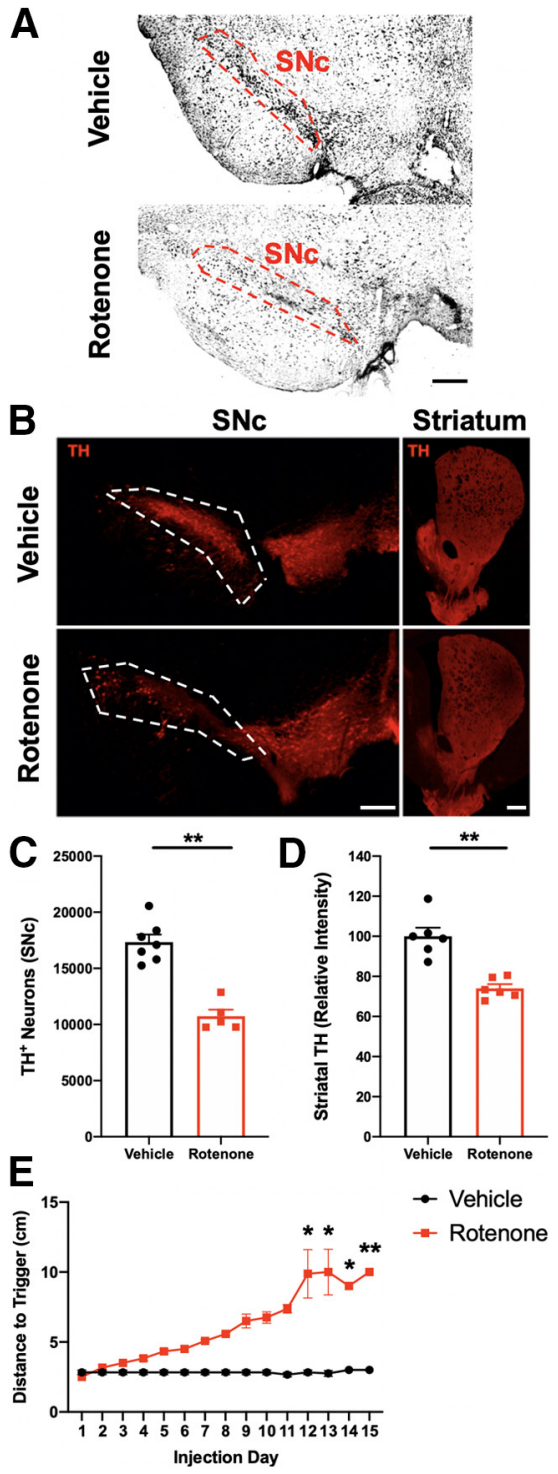

Figure 1. Rotenone-induced nigrostriatal degeneration causes motor deficits in adult rats. A, Representative images of Nissl-stained midbrain from rotenone- or vehicle-treated rats, indicating substantial neuronal cell body loss in the SNc in response to rotenone; $20 \times$ magnification. Dashed lines indicate SNc. Scale bar, $100 \mu \mathrm{m}$. $\boldsymbol{B}$, Representative images of TH-immunostained DA neurons (red) from the SNc (left) and striatum (right) from vehicle- or rotenone-treated rats; $20 \times$ magnification. Dashed lines indicate SNc. Scale bars, $100 \mu \mathrm{m}$. C, Quantification of immunostained $\mathrm{TH}^{+}$DA neurons in the SNc (Mann-Whitney $U$ test, $p=0.0025)$ and $(\boldsymbol{D}) \mathrm{TH}$ fluorescence intensity from $\mathrm{TH}^{+}$nerve terminals in the dorsolateral striatum showed significant loss in response to rotenone compared with vehicle (MannWhitney $U$ test, $p=0.0022$ ). Striatal TH fluorescence intensity is normalized to vehicle condition. $\boldsymbol{E}$, PIT of vehicle (black)- or rotenone (red)- treated rats shows impairment in rotenonetreated rats $\left(\mathrm{H}_{14}=41.8, p<0.001\right)$. Data are mean \pm SEM. $N=5-7$ per group for stereology and immunohistochemistry. $N=3$ per group for PIT. ${ }^{*} p<0.05 .{ }^{* *} p<0.01$.

significantly worse performance on the test starting at day 12 compared with day 1 of drug administration (post hoc $p=0.015$ ). Together, the combined decreases in Nissl staining and TH immunoreactivity in the SNc and striatum, coupled with these PIT data, indicate that our rotenone treatment successfully produced substantial midbrain DA neuron loss over time.

\section{VGLUT2-expressing DA neurons are protected from} rotenone-induced neurodegeneration To investigate rotenone's effect on midbrain TH and VGLUT2 expression, TH and VGLUT2 mRNA levels were measured in 
A

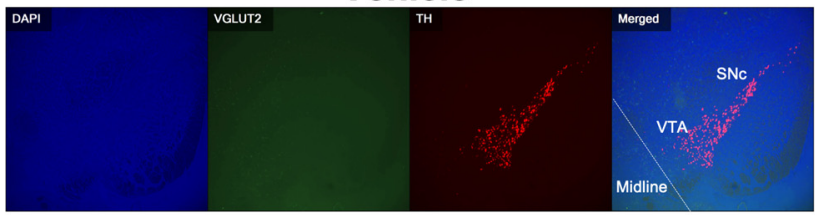

B

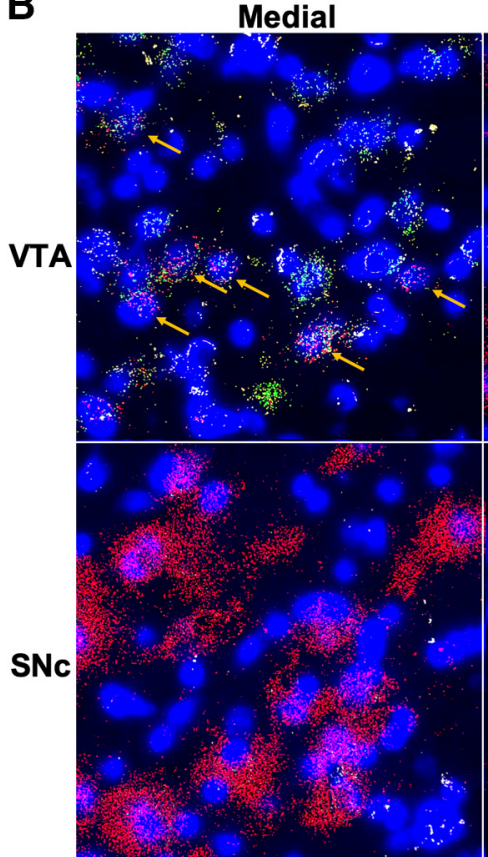

C

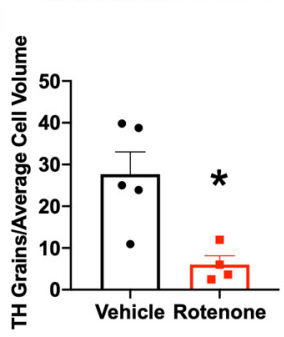

D

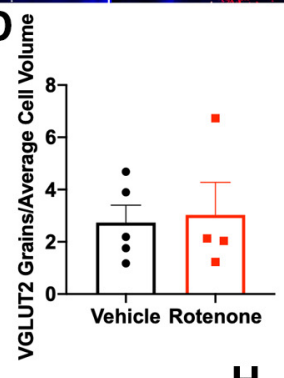

G

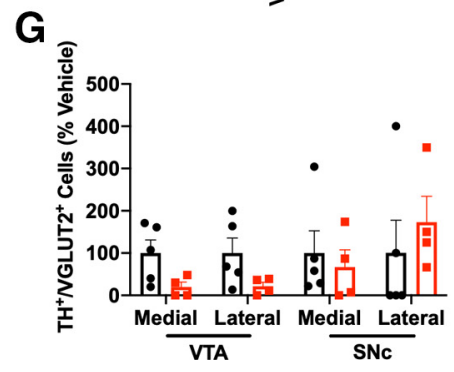

Lateral

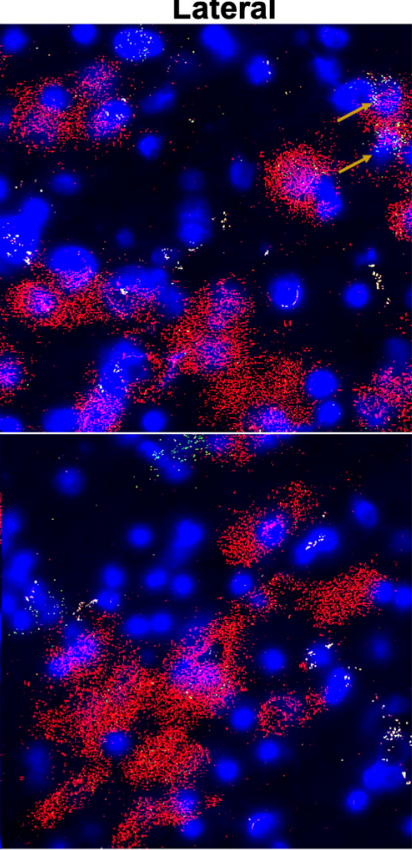

E
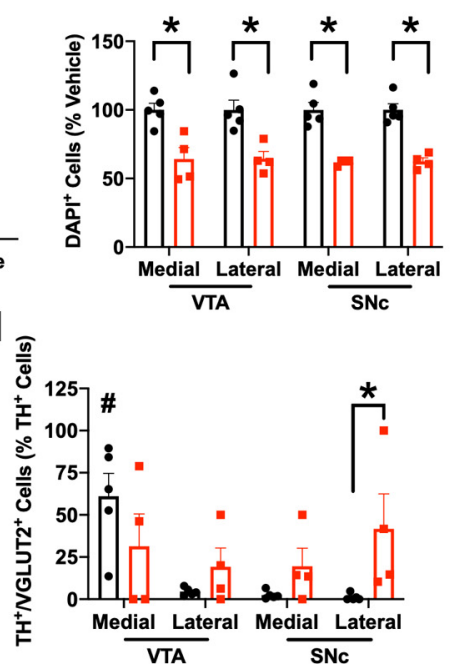

Rotenone

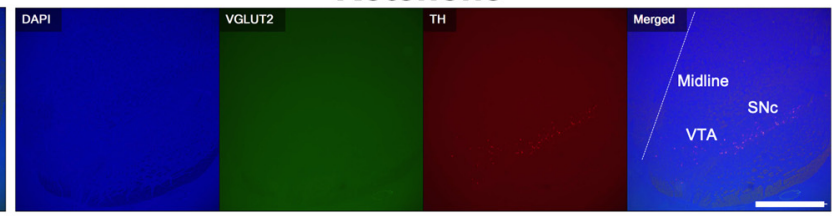

Medial
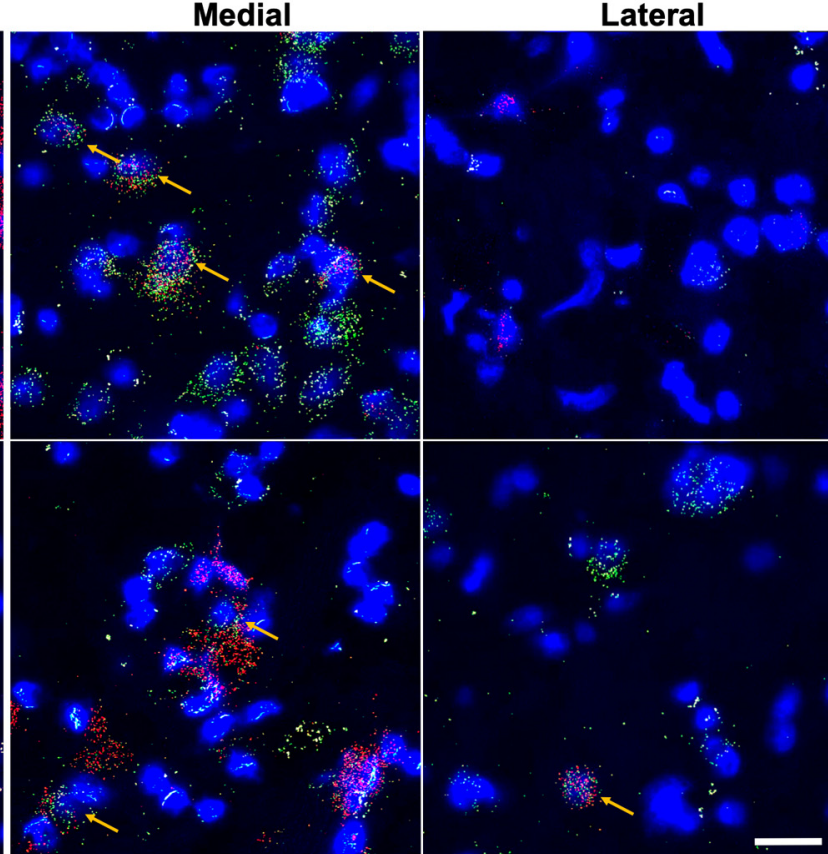

$\mathbf{F}$

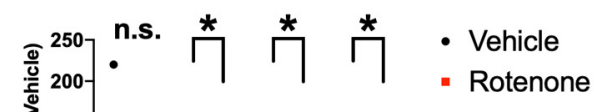

Figure 2. VGLUT2-expressing DA neurons are more resistant to rotenone-induced cell loss. Representative images of TH and VGLUT2 mRNA in vehicle- and rotenone-treated rats. $A$, $4 \times$ and (B) $60 \times$ magnification images of VTA/SNc of vehicle-treated rats showed a high level of TH expression (red) and a subset of TH ${ }^{+}$cells that coexpress VGLUT2 (green). Yellow arrows indicate $\mathrm{TH}^{+} /$NGLUT2 $^{+}$-coexpressing cells. Scale bars: $4 \times$ image, $2 \mathrm{~mm} ; 60 \times$ image, $25 \mu \mathrm{m}$. Lipofuscin depicted in $60 \times$ images (white). C-I, Quantification of TH and VGLUT2 mRNA expression in rotenone- and vehicle-treated rats. $\boldsymbol{C}, \boldsymbol{D}$, Although TH mRNA grains per average cell volume were significantly decreased in rotenone-treated rats compared with vehicle (Mann-Whitney $U$ test, $p=0.032 ; \boldsymbol{C}$, there was no change in VGLUT2 grains per average cell volume in rotenone-treated rats (Mann-Whitney $U$ test, $p=0.99 ; \boldsymbol{D}$ ). $\boldsymbol{E}$, There was a significant decrease in number of DAPI-stained nuclei in VTA and SNc in response to rotenone treatment. $\boldsymbol{F}$, Rotenone diminished the number of $\mathrm{TH}^{+}$cells in both VTA and SNc compared with controls. $\boldsymbol{G}$, In contrast, $\mathrm{TH}^{+} /$ VGLUT2 $^{+}$cell numbers were not significantly changed with rotenone treatment. $\boldsymbol{H}$, The percentage of $\mathrm{TH}^{+}$cells that expressed VGLUT2 increased after rotenone treatment, indicating that TH/ VGLUT2-coexpressing neurons were more resistant to rotenone-induced neurodegeneration. $I$, There was no increase in VGLUT2 expression within $\mathrm{TH}^{+} / \mathrm{NGLUT2}^{+}$cells after rotenone. Data are mean \pm SEM. $N=4$ or 5 per group. ${ }^{*} p<0.05$ compared with vehicle. ${ }^{\sharp} p<0.05$ compared with other regions.

the VTA and SNc of vehicle- and rotenone-treated rats via multiplex RNAscope (Fig. 2). Rotenone significantly decreased overall TH mRNA expression in the ventral midbrain, which was measured as the number of grains per average cell volume in an image
$(U=1, p=0.032$; Fig. $2 C)$. In contrast, rotenone did not change VGLUT2 mRNA expression $(U=10, p>0.99$; Fig. $2 D)$. We also examined effects of rotenone on DAPI-labeled cell density in the VTA and $\mathrm{SNc}$ and discovered that rotenone diminished 
A

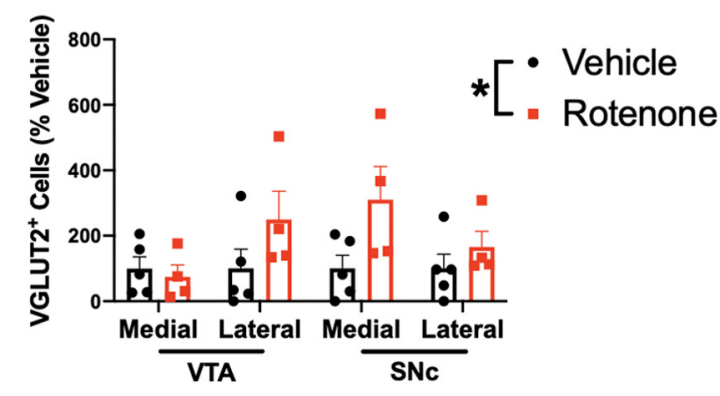

B

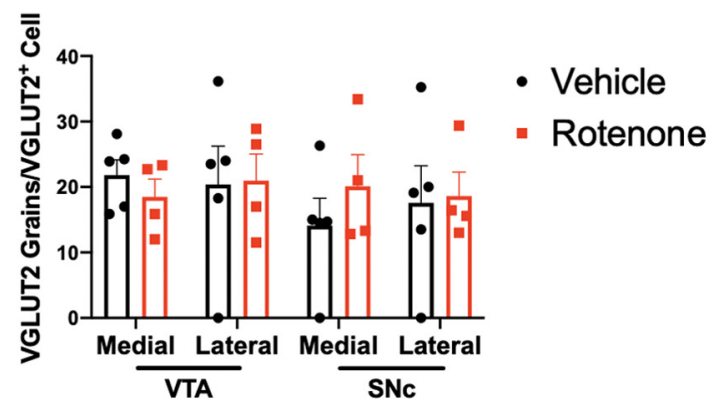

Figure 3. Rotenone increases density of VGLUT2 ${ }^{+}$neurons. $A$, Rotenone treatment increased the total number of VGLUT2 ${ }^{+}$cells that did not also coexpress $\mathrm{TH}_{\mathrm{mRNA}}\left(\mathrm{TH}^{-} / \mathrm{VGLUT2}^{+}\right.$) $\left(\mathrm{H}_{1}\right.$ $=4.6, p=0.033)$. $B$, There was no change in VGLUT2 mRNA grain expression within $\mathrm{TH}^{-} / \mathrm{NGLUT2}^{+}$cells in either VTA or SNc of rotenone-treated rats compared with the vehicle control. Data are mean \pm SEM. $N=4$ or 5 per group, ${ }^{*} p<0.05$.

numbers of DAPI-stained nuclei $\left(\mathrm{H}_{1}=25.6, p<0.001\right)$, with no differences in this decrease between the VTA and $\mathrm{SNc}\left(\mathrm{H}_{3}=\right.$ $0.03, p>0.99$; Fig. $2 E$ ). Both the VTA and SNc had a significantly lower number of $\mathrm{DAPI}^{+}$cells $(40 \%$ reduction) after rotenone treatment compared with vehicle (post hoc $p<0.05$ for all regions). These findings strongly suggest that rotenone caused cell death in the ventral midbrain, confirming our initial observations (Fig. 1) and consistent with earlier work (Cannon et al., 2009; Blesa and Przedborski, 2014; Johnson and Bobrovskaya, 2015; Radad et al., 2019). Rotenone also led to a severe reduction $(>85 \%)$ of DA neurons expressing only $\mathrm{TH}\left(\mathrm{TH}^{+}, \mathrm{H}_{1}=22.2\right.$, $p<0.001$; Fig. $2 F$ ). Notably, there was a relative sparing of the medial VTA, which demonstrated a smaller reduction $(60 \%)$ in TH-expressing DA cells $(p=0.29)$.

Midbrain DA neurons that also expressed VGLUT2 $\left(\mathrm{TH}^{+} /\right.$ VGLUT2 $^{+}$) did not exhibit significant cell number decreases in response to rotenone $\left(\mathrm{H}_{1}=1.1, p=0.28\right.$; Fig. $\left.2 G\right)$, suggesting that $\mathrm{TH}^{+} / \mathrm{VGLUT2}^{+}$neurons were more resilient to rotenone compared with DA neurons that only expressed TH. When compared as a percentage of all $\mathrm{TH}^{+}$cells, a higher percentage of $\mathrm{TH}^{+}$cells were VGLUT2 ${ }^{+}$after rotenone treatment compared with vehicle in the lateral SNc $(p=0.048$; Fig. $2 H)$. Additionally, in vehicle-treated animals, there was a regional difference in the proportion of $\mathrm{TH}^{+}$cells that were also VGLUT2 ${ }^{+}$. We found that the medial VTA had the highest percentage of $\mathrm{TH}^{+}$cells that were $\mathrm{VGLUT}^{+}{ }^{+}$compared with all other brain regions (post hoc $p=0.024$ vs lateral VTA, medial SNc, and lateral SNc; Fig. $2 \mathrm{H}$ ), consistent with previous studies (Yamaguchi et al., 2011, 2015). Finally, we investigated whether the number of VGLUT2 grains within $\mathrm{TH}^{+} / \mathrm{VGLUT}^{+}$neurons was altered by rotenone. We found neither a significant effect of rotenone $\left(\mathrm{H}_{1}=0.5\right.$, $p=0.50)$ nor regional differences $\left(\mathrm{H}_{3}=2.7, p=0.43\right.$; Fig. $\left.2 I\right)$. This indicates that, while $\mathrm{TH}^{+} / \mathrm{VGLUT}^{+}$cells were more likely to survive, rotenone exposure did not change levels of VGLUT2 mRNA expression in midbrain DA neurons. In all, our findings suggest that neurons in the medial VTA are relatively spared from rotenone-induced degeneration, and that VGLUT2 expression in DA neurons confers increased resilience to rotenoneinduced DA neurodegeneration in the midbrain.

\section{Rotenone increases density of VGLUT2 ${ }^{+}$cells negative for} TH in the VTA and SNc

Surprisingly, after rotenone treatment, we found an increase in the density of midbrain cells that were VGLUT2 ${ }^{+}$but that did not coexpress TH mRNA compared with vehicle-treated animals $\left(\mathrm{H}_{1}\right.$ $=4.6, p=0.033$; Fig. $3 A$ ). There was no significant effect of rotenone $\left(\mathrm{H}_{1}=0.1, p=0.82\right)$ or brain region $\left(\mathrm{H}_{3}=2.1, p=0.55\right)$ on VGLUT2 mRNA grain numbers within VGLUT2 ${ }^{+}$cells (Fig. $3 B$ ). These findings indicate that rotenone treatment increases the number of cells with detectable VGLUT2 expression while not increasing the total amounts of VGLUT2 mRNA within these VGLUT2 $^{+}$cells.

\section{Rotenone increases VGLUT2 protein expression in the VTA and $\mathrm{SNc}$}

Since midbrain DA neurons that expressed VGLUT2 at the mRNA level were protected from rotenone-induced neurodegeneration, we investigated whether these results also translated to the level of VGLUT2 protein expression (Fig. 4). There was no significant effect of rotenone $\left(\mathrm{H}_{1}=2.8, p=0.09\right)$ or brain region (i.e., VTA vs $\mathrm{SNc})$ on VGLUT2 puncta intensity $\left(\mathrm{H}_{1}=0.0\right.$, $p=1.0$; Fig. $4 C$ ). In contrast, we found significant effects of rotenone $\left(\mathrm{H}_{1}=6.7, p=0.01\right)$ and brain region $\left(\mathrm{H}_{1}=3.9, p=0.049\right)$ on the density of VGLUT2 $^{+}$puncta, indicating an increase in glutamatergic terminals projecting into the midbrain (Fig. 4D).

Surprisingly, we observed a main effect of rotenone $\left(\mathrm{H}_{1}=7.6\right.$, $p=0.006)$ on VGLUT2 puncta within midbrain TH-immunoreactive cells (Fig. $4 E$ ), suggesting rotenone-induced upregulation of VGLUT2 protein in DA cell bodies. Since significant quantities of VGLUT2 puncta have not been typically reported in the cell bodies of DA neurons under physiological conditions (Hnasko et al., 2010; Fortin et al., 2019; Silm et al., 2019), we examined VGLUT2 localization in rotenone-treated N27 cells, immortalized rat mesencephalic dopaminergic cells that share key features of DA neurons and that also endogenously express VGLUT2 (Clarkson et al., 1998; Drechsel et al., 2007). We found that $24 \mathrm{~h}$ treatment with rotenone (100 $\mathrm{nm}$ ) produced significant cortical $\beta$-actin reorganization accompanied by retraction of dopaminergic cell processes, including axons (Fig. 5). In the absence of efficient trafficking to the axons because of rotenone-induced changes to the cytoskeleton, these results suggest a mechanism by which newly produced VGLUT2 accumulates in the cell bodies of DA neurons in response to rotenone.

\section{Striatal VGLUT2 ${ }^{+}$DA terminals are more resilient to rotenone treatment}

We investigated whether rotenone treatment altered VGLUT2 expression in the nerve terminals projecting to the striatum from midbrain DA neurons (Fig. 6A). We specifically compared $\mathrm{TH}$ and VGLUT2 protein expression in striatal DA nerve terminals within the $\mathrm{CPu}$ and the NAc, as DA neurons in the SNc largely 
A
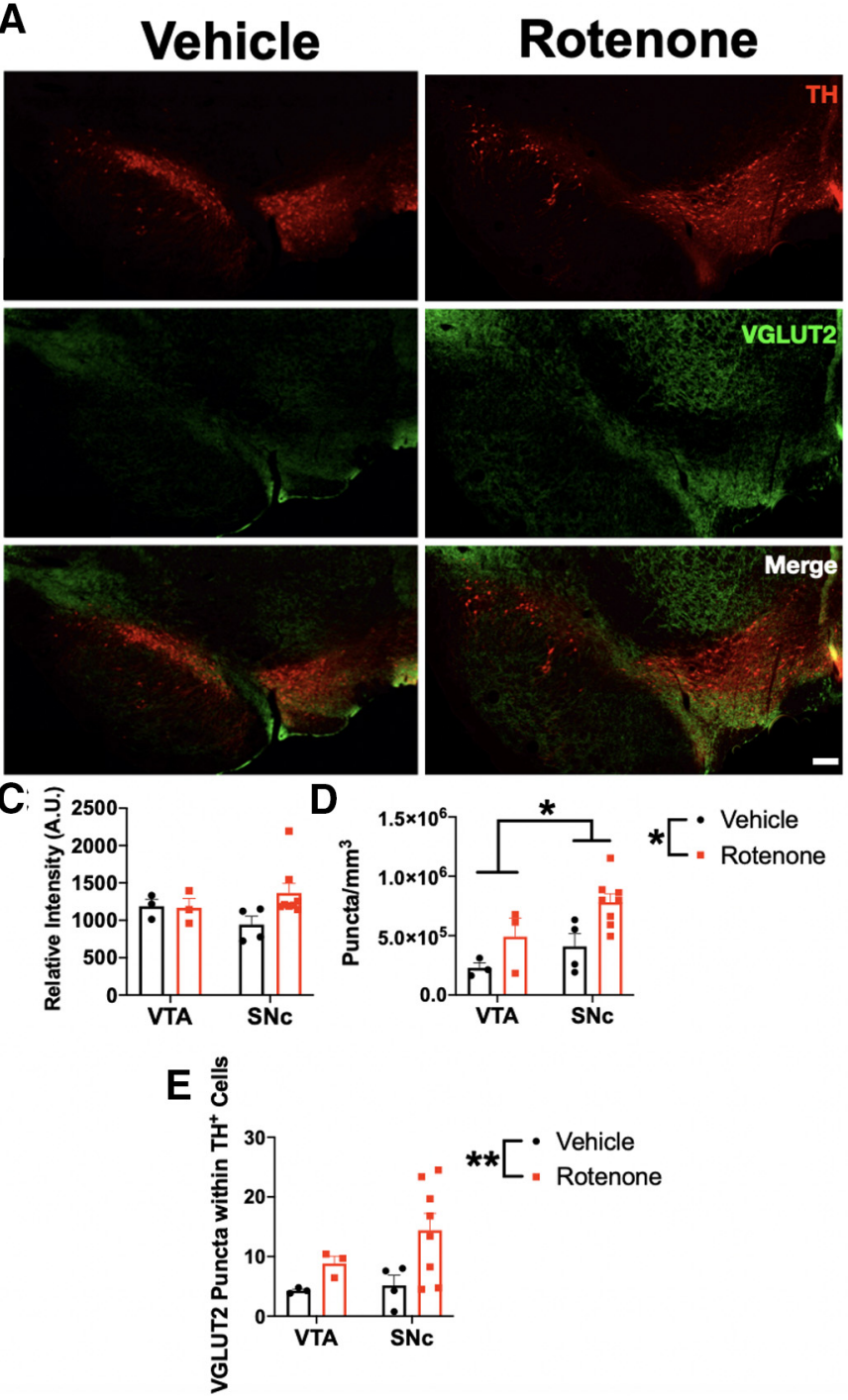

B

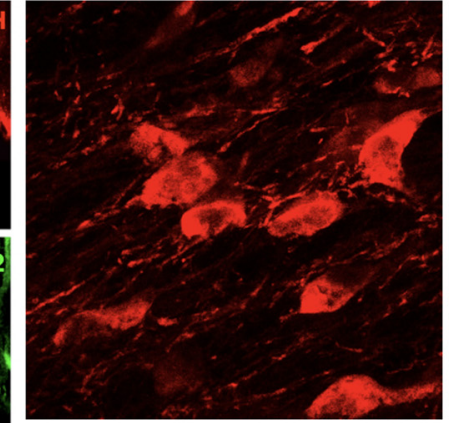

Vehicle
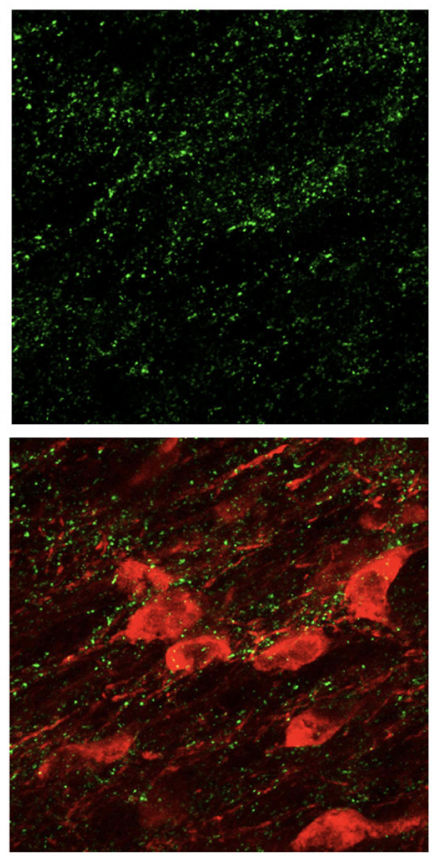

Rotenone

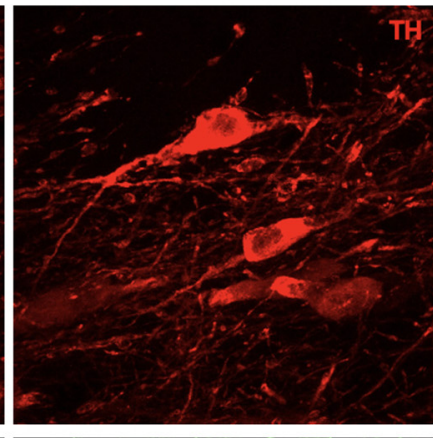

VGLUT2
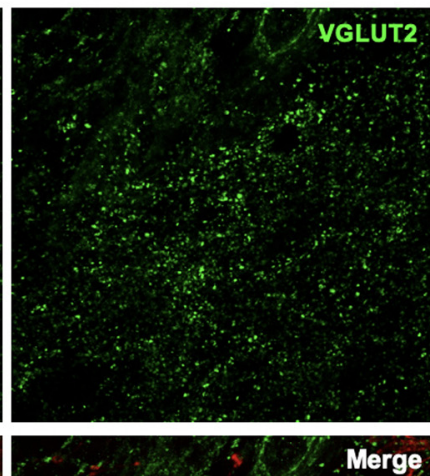

Figure 4. Rotenone upregulates VGLUT2 protein expression in surviving DA neurons. $\boldsymbol{A}$, Representative $20 \times$ and $(\boldsymbol{B}) 100 \times$ images showing an upregulation of VGLUT2 in the VTA and SNc of rotenone-treated rats. Scale bar, $30 \mu \mathrm{m}$. C, While there was no significant change in relative intensity of VGLUT2 ${ }^{+}$puncta in either the VTA or SNc, there was a significant increase in (D) VGLUT2 $^{+}$puncta density and $(\boldsymbol{E})$ the number of VGLUT2-immunoreactive puncta within $\mathrm{TH}^{+}$cells. Data are mean \pm SEM. $N=3-8$ per group. ${ }^{*} p<0.05 .{ }^{*} p<0.01$.

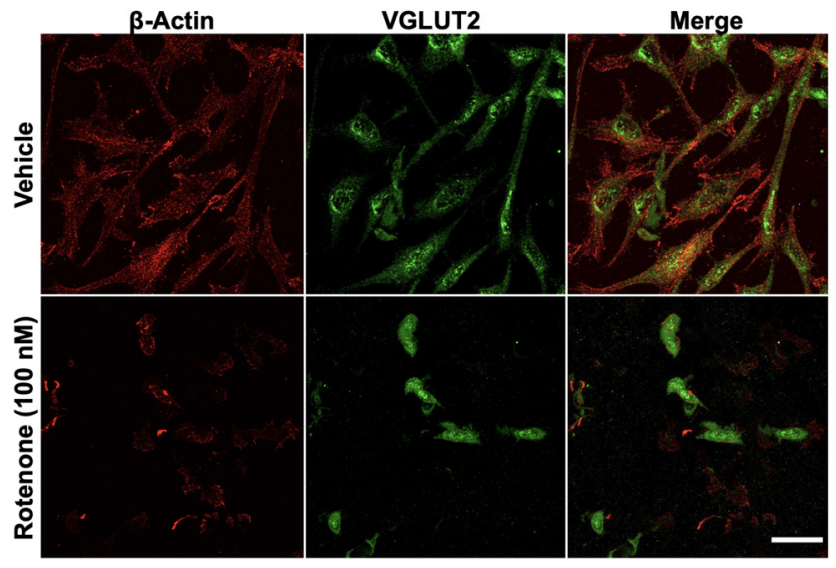

Figure 5. Rotenone treatment alters actin cytoskeleton and VGLUT2 distribution in N27 cells. Representative images of the rat dopaminergic neuron N27 cell line showed increased VGLUT2 signal (green) in the cell body with accompanying retraction of cell processes and reorganization of cortical $\beta$-actin (red) after $100 \mathrm{~nm}$ rotenone treatment. Scale bar, $20 \mu \mathrm{m}$. project to the $\mathrm{CPu}$ (Gibb, 1992; Abeliovich et al., 2000; Matsuda et al., 2009; Sulzer and Surmeier, 2013; Surmeier et al., 2017), whereas DA neurons in the VTA project to the NAc (Ikemoto, 2010; Mingote et al., 2019). Moreover, in examining the NAc projections, we split our analyses according to the anatomically well-defined NAc shell and core subregions, as DA neurons in medial VTA send a significant number of projections to the NAc shell, whereas those in the lateral VTA project to the NAc core (Yamaguchi et al., 2011; Mingote et al., 2015, 2017, 2019; Poulin et al., 2018). There was a regional difference in intensity of $\mathrm{TH}$ immunoreactivity, suggesting region-specific differences in $\mathrm{TH}$ expression $\left(\mathrm{H}_{2}=6.4, p=0.041\right.$; Fig. $\left.6 \mathrm{~B}\right)$. Analysis of specific subregions revealed significantly decreased $\mathrm{TH}$ signal intensity in the $\mathrm{CPu}$ (post hoc $p=0.048$ ) but not in the NAc core (post hoc $p>0.99$ ) or shell (post hoc $p>0.99$ ). This suggests that expression of TH is more vulnerable to rotenone in DA terminals projecting to the $\mathrm{CPu}$ compared with terminals in the NAc. Next, we examined whether rotenone altered the density of DA terminals projecting to the striatum. Quantification of the $\mathrm{TH}^{+}$ puncta representing these DA nerve terminals in the $\mathrm{CPu}$ versus NAc subregions (core, shell) showed a regional difference in the 
density of $\mathrm{TH}^{+}$striatal DA terminals $\left(\mathrm{H}_{2}=12.5, p=0.002\right.$; Fig. $\left.6 B\right)$. We found an overall greater density of $\mathrm{TH}^{+}$terminals in the $\mathrm{CPu}$ compared with the NAc core $(p=0.006)$. Moreover, although there was no significant main effect of rotenone $\left(\mathrm{H}_{1}=0.27, p=0.61\right)$, post hoc comparisons revealed decreased $\mathrm{TH}^{+}$ terminal density in the $\mathrm{CPu}$ after rotenone treatment $(p=0.048)$, suggesting that $\mathrm{TH}^{+}$terminals in the $\mathrm{CPu}$ are more vulnerable to rotenone versus those in the NAc shell or core. Together, these findings suggest regional differences in resilience to rotenone within the striatum, with NAc terminals more resilient to rotenone-induced decreases in $\mathrm{TH}$ expression and terminal density compared with $\mathrm{CPu}$ terminals.

We also examined striatal VGLUT2, finding neither significant differences in signal intensity nor puncta density (all $p>0.06$; Fig. 6C). This suggests that, unlike in the VTA and SNc, VGLUT2 protein expression and axonal integrity are unaltered throughout the striatum after rotenone administration.

Finally, we analyzed the density of the subpopulation of DA terminals that express both TH and VGLUT2 in striatum. We found a greater density of $\mathrm{TH}$ puncta that colocalized with VGLUT2 in the NAc shell compared with NAc core $\left(\mathrm{H}_{1}=4.7, p=0.031\right.$; Fig. $\left.6 D, E\right)$. This is consistent with recent work showing that medial VTA DA/glutamate neurons preferentially innervate the NAc shell (Yamaguchi et al., 2011; Mingote et al., 2015, 2017, 2019; Poulin et al., 2018). Further, comparing TH/VGLUT2 pixel colocalization to randomized $\mathrm{TH}$ and VGLUT2 pixel distributions in the same images confirmed that colocalization levels of $\mathrm{TH}^{+}$and $\mathrm{VGLUT2}^{+}$puncta in the NAc were greater than by random chance (Extended Data Fig. 6-1). However, rotenone treatment did not increase TH and VGLUT2 colocalization in the NAc $\left(\mathrm{H}_{1}=3.5, p=0.061\right.$; Fig. 6E), suggesting that VGLUT2 expression in striatal DA terminals was not significantly altered.

\section{Discussion}

We used the rotenone model of PD to examine VGLUT2-dependent mechanisms of selective DA neuron vulnerability since, in contrast to many DA neurotoxin models (e.g., 6-OHDA, MPTP), rotenone recapitulates critical neuropathological features of $\mathrm{PD}$, including endolysosomal dysfunction, neuroinflammation, and $\alpha$-synuclein accu-

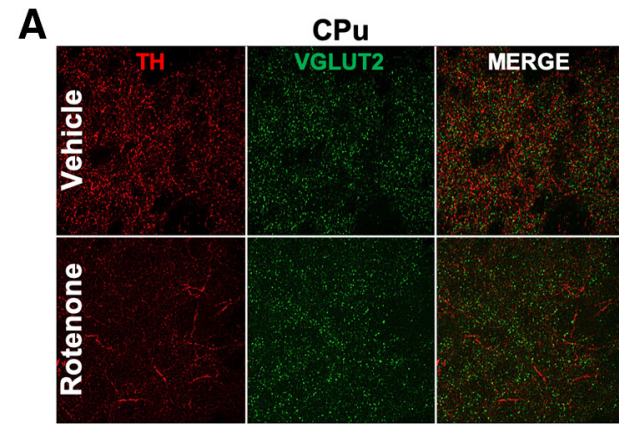

A
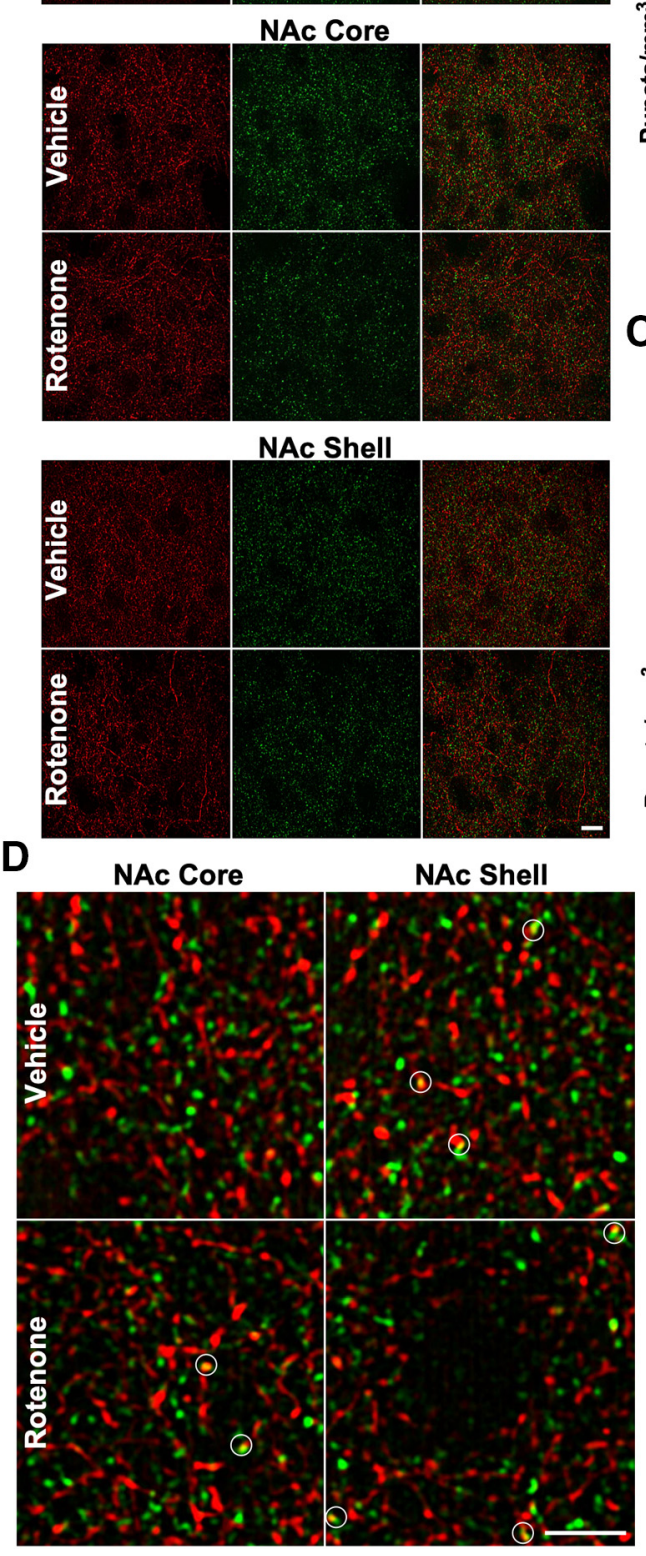

B

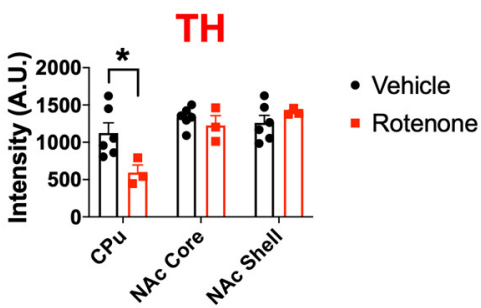

C VGLUT2
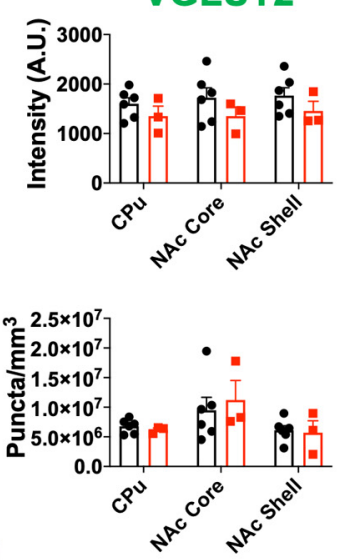

E

TH/VGLUT2 Overlap

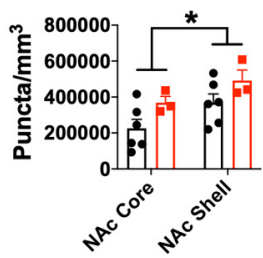

Figure 6. Rotenone leads to region-specific loss of TH protein and increased density of TH and VGLUT2-colocalizing puncta in the NAc. $\boldsymbol{A}$, Representative $60 \times$ images showing loss of TH signal but no change in VGLUT2 in the CPu of rotenone-treated rats. Scale bar, $20 \mu \mathrm{m}$. B. There was a significantly lower TH signal intensity in the CPu (Dunn's post hoc $p=0.048$ ), but not the NAc core (post hoc $p>0.99$ ) or shell (post hoc $p>0.99$ ), after rotenone treatment. Similarly, there was a significantly lower TH puncta density in the CPu (post hoc $p=0.048$ ), but not in the NAc core (post hoc $p>0.99$ ) or shell (post hoc $p>0.99$ ), after rotenone treatment. $C$, There was no change in the intensity or density of VGLUT2 ${ }^{+}$puncta in nerve terminals within the CPu or NAc of rotenone-treated rats compared with vehicle (Kruskal-Wallis H test, all $p>0.06$ ). D, Representative $60 \times$ images of TH (red) and VGLUT2 (green) puncta in the NAC core and shell in vehicle- and rotenone-treated rats. White circles represent colocalizing TH and VGLUT2 puncta. Scale bar, $10 \mu \mathrm{m}$. $E$, Within the NAc, a greater density of TH puncta colocalized with VGLUT2 in the NAc shell compared with the NAC core (Kruskal-Wallis $\mathrm{H}$ test, $p=0.031$ ), but there was no significant effect of rotenone $(p=0.061)$. Data are mean \pm SEM. $N=3-6$ per group. ${ }^{*} p<0.05$ compared with vehicle. ${ }^{\#} p<0.05$ compared with CPu. See Extended Data Figure 6-1. 
mulation (Johnson and Bobrovskaya, 2015). We found that chronic rotenone administration decreased DA neuron number, consistent with prior stereological counts of $\mathrm{TH}^{+} / \mathrm{Nissl}^{+} \mathrm{DA}$ neurons after rotenone (Cannon et al., 2009; De Miranda et al., 2019). Although rotenone-induced cell loss was less severe versus acute models, such as 6OHDA (Betarbet et al., 2002), it was still sufficient to impair motor performance. This may be since rotenone is more focused than other DA neurotoxins by producing striatal DA terminal loss specifically in the dorsolateral striatum (Cannon et al., 2009). Additionally, because rotenone is a systemic toxicant, it may take longer to cause profound DA neurodegeneration than other toxins, which are injected directly into brain (Martinez and Greenamyre, 2012). Finally, most studies correlating motor function with DA neurodegeneration have relied on neurotoxin-induced unilateral lesions. However, compensation involving the unlesioned hemisphere likely masks motor impairment and raises levels of nigral cell loss needed to detect motor deficits. In contrast, rotenone causes simultaneous, bilateral nigrostriatal lesions that limit compensation, enabling detection of motor dysfunction at lesser levels of nigral cell loss.

We found that rotenone-induced decreases in DA neuron number were greater than decreases in $\mathrm{TH}$ immunoreactivity in the dorsolateral striatum. This is consistent with some reports (Tapias et al., 2014, 2019) but not others (Betarbet et al., 2000; De Miranda et al., 2019), likely because of variability in rotenone's severity between cohorts and the appearance of hypertrophic TH terminals because of compensation (Cannon et al., 2009). We also observed greater loss of TH mRNA compared with $\mathrm{TH}$ protein. DA neurons may compensate for rotenoneinduced decreases in TH mRNA expression by upregulating translation and/or inhibiting degradation of $\mathrm{TH}$ protein to maintain normal levels of the enzyme. Second, TH protein has a halflife $(\sim 30 \mathrm{~h})$ approximately double that of TH mRNA (Tank et al., 1986; Roe et al., 2004), suggesting that loss of TH protein is slower than mRNA. Ultimately, severe loss of TH mRNA because of rotenone may obscure regional differences in vulnerability between VTA and SNc earlier in the degenerative process, although medial VTA $\mathrm{TH}^{+}$cells are still relatively protected.

$\mathrm{TH}^{+} / \mathrm{VGLUT}^{+}$cells are enriched in the medial VTA and project to the medial NAc shell (Poulin et al., 2018; Mingote et al., 2019). Importantly, medial VTA DA neurons and their striatal projections are selectively protected from neurodegeneration compared with $\mathrm{SNc}$, raising the possibility that this VGLUT2 $^{+}$subpopulation is more resilient to rotenone (Surmeier et al., 2017). Indeed, we observed resilience of dopaminergic terminals in the NAc shell, which are predominantly $\mathrm{TH}^{+} / \mathrm{VGLUT2}^{+}$(Mingote et al., 2019). However, medial VTA $\mathrm{TH}^{+} / \mathrm{VGLUT2}^{+}$neurons appeared to be less protected than $\mathrm{TH}^{+} / \mathrm{VGLUT2}^{+}$neurons in other midbrain regions. Because medial VTA DA neurons that did not express VGLUT2 were also protected from rotenone, we propose that the higher survival rates for all medial $\mathrm{VTA} \mathrm{TH}^{+}$neurons may mask the greater protection $\mathrm{TH}^{+} / \mathrm{VGLUT}^{+}$neurons enjoy in other midbrain regions. Alternatively, $\mathrm{VGLUT}^{+} \mathrm{DA}$ neurons may belong to a larger group of medial VTA neurons protected via other mechanisms. Earlier studies demonstrated that other subpopulations of $\mathrm{TH}^{+}$cells in the medial VTA are similarly protected in PD models, including $\mathrm{TH}^{+}$cells that express calbindin (Liang et al., 1996; Poulin et al., 2014, 2018). Previous work suggests calbindin's ability to buffer calcium protects mitochondria from PD neurotoxins (Jung et al., 2019; Ricke et al., 2020). When mitochondrial respiratory complexes are inhibited, calcium levels rise and enhance mitochondrial reactive oxygen species (ROS) production (Feissner et al., 2009; Freestone et al., 2009). By diminishing local calcium, calbindin's calcium buffering may decrease ROS and boost resilience (Brookes et al., 2004; Feissner et al., 2009). Interestingly, $\mathrm{TH}^{+} /$ VGLUT2 $^{+}$neurons in the lateral SNc exhibited the greatest protection from rotenone, suggesting that these neurons express additional factors that further enhance resilience. Indeed, lateral SNc $\mathrm{TH}^{+} /$VGLUT2 $^{+}$neurons possess expression patterns distinct from medial VTA $\mathrm{TH}^{+} /$VGLUT2 $^{+}$neurons (Poulin et al., 2018). Future studies will identify these subpopulation-specific markers that also modify DA neuron resilience.

We observed increased VGLUT2 protein within DA neuron cell bodies in response to rotenone. While VGLUT2 trafficking to nerve terminals is well established, its localization to the soma is less understood. Elevated levels of VGLUT2 protein in the cell bodies may be the consequence of rotenone-induced disruptions in protein trafficking from the soma to the cell periphery. Consistent with this, we found retraction of dopaminergic cell processes likely because of alterations in actin cytoskeletal organization. Indeed, $\alpha$-synuclein-positive inclusions have been found in DA neurons after rotenone treatment which impair intracellular trafficking as well as endoplasmic reticulum and Golgi apparatus function (Betarbet et al., 2000; Mazzulli et al., 2016). It is therefore possible that a novel mechanism of rotenone's cytotoxicity is because of impaired ability of DA neurons to transport VGLUT2 $^{+}$vesicles to the axon terminals for release.

Surprisingly, we observed altered VGLUT2 protein expression in $\mathrm{TH}^{+}$cell bodies despite absence of significant changes in VGLUT2 mRNA expression. This lack of rotenone-induced DA neuron VGLUT2 mRNA upregulation contrasts with upregulation of DA neuron VGLUT2 mRNA levels previously described in response to 6-OHDA and MPTP (Dal Bo et al., 2008; Shen et al., 2018; Steinkellner et al., 2018; Kouwenhoven et al., 2020). Unlike these acute toxins, since rotenone induces its neurotoxicity over a subchronic timeframe, we cannot exclude that VGLUT2 mRNA expression may increase as an early response to neuronal damage only to recede later and therefore not be captured by the study endpoint. Additionally, while an overall low proportion of $\mathrm{TH}^{+}$ terminals colocalize with VGLUT2 across the entire NAc, there are distinct NAc subregions exhibiting more TH and VGLUT2 colocalization than others, suggesting spatially segregated sites particularly enriched in glutamatergic vesicles within DA axons and terminals (Fortin et al., 2019; Silm et al., 2019).

There was an overall increase in cells expressing VGLUT2 mRNA in the VTA and SNc after rotenone. This was accompanied by an increase in VGLUT2 ${ }^{+}$terminal density in the VTA and $\mathrm{SNc}$, suggesting that rotenone also acts on nondopaminergic cells. Consistent with this, the VTA possesses glutamatergic afferents that express VGLUT2 (Geisler and Wise, 2008). Intriguingly, genetic fate-mapping demonstrated that, like DA neurons, the majority of cholinergic neurons in the mesopontine tegmentum also express VGLUT2 during development only to repress it in adulthood; these cholinergic neurons include the pedunculopontine tegmental nucleus, which projects to the midbrain (Steinkellner et al., 2019). Thus, neurotoxin exposure may lead to reemergence of VGLUT2 expression in adult pedunculopontine tegmental nucleus neurons that project to midbrain as part of a neuroprotective response (Steinkellner et al., 2018, 2019). It is also possible that some $\mathrm{TH}^{-} / \mathrm{VGLUT}^{+}{ }^{+}$neurons were DA neurons that downregulated $\mathrm{TH}$ after rotenone exposure. Earlier studies showed chronic rotenone decreased $\mathrm{TH}$ protein expression in rodent midbrain (Sherer et al., 2003; Testa et al., 2005). Such TH downregulation may boost resilience, as decreasing DA synthesis via diminished 
TH expression could lower ROS-generating reactive DA metabolites (e.g., DOPA quinones) (Asanuma et al., 2003).

Our data suggest that VGLUT2 expressed in midbrain boosts resilience to rotenone, but the mechanisms remain poorly characterized. We previously demonstrated that VGLUT2 dynamically enhances activity-dependent vesicular DA loading and release (Aguilar et al., 2017), suggesting that DA neuron VGLUT2 may be used by surviving neurons to maintain synaptic DA neurotransmission. By increasing DA sequestration into vesicles, VGLUT2 may diminish the cytoplasmic DA available for conversion into ROS (Meiser et al., 2013). Last, VGLUT2 may modulate local glutamate availability to mitochondria to protect neurons from metabolic dysfunction after insults. In addition to being an excitatory neurotransmitter, neurons use glutamate as an anaplerotic energy source. Glutamate is converted to $\alpha$-ketoglutarate to fuel the TCA cycle and maintain adequate ATP synthesis, which may help restore metabolic homeostasis (Divakaruni et al., 2017).

Study limitations include the inability to distinguish between purely glutamatergic VGLUT2 $^{+}$midbrain neurons versus VGLUT2 $^{+}$dopaminergic neurons that substantially downregulated $\mathrm{TH}$ in response to rotenone toxicity. Future work needs to identify markers distinct for nondopaminergic VGLUT2 ${ }^{+}$neurons and those unique to $\mathrm{TH}^{+} / \mathrm{VGLUT}^{+}$cells. Second, limitations of light microscopy make it difficult to definitively resolve the extent to which VGLUT2 ${ }^{+}$puncta localize to dopaminergic terminals and whether rotenone treatment alters this distribution. Follow-up studies using electron- and super-resolution microscopy can conclusively determine the localization of VGLUT2 ${ }^{+}$ puncta. Finally, we did not investigate sex differences since the rotenone dosage that causes severe DA neurodegeneration in males does not cause degeneration in females (De Miranda et al., 2019). Subsequent studies will examine other toxicants that cause degeneration in both sexes at identical doses to directly compare males versus females.

In conclusion, we show that $\mathrm{TH}^{+} / \mathrm{VGLUT2}^{+}$cells are more resilient to rotenone and that rotenone-induced VGLUT2 upregulation throughout the VTA and SNc may represent a neuroprotective mechanism, shielding neurons from neurodegeneration. Elucidating mechanisms behind VGLUT2-mediated neuroprotection may lead to novel therapeutic strategies to mitigate or prevent DA neuron neurodegeneration.

\section{References}

Abeliovich A, Schmitz Y, Farinas I, Choi-Lundberg D, Ho WH, Castillo PE, Shinsky N, Verdugo JM, Armanini M, Ryan A, Hynes M, Phillips H, Sulzer D, Rosenthal A (2000) Mice lacking alpha-synuclein display functional deficits in the nigrostriatal dopamine system. Neuron 25:239-252.

Aguilar JI, Dunn M, Mingote S, Karam CS, Farino ZJ, Sonders MS, Choi SJ, Grygoruk A, Zhang Y, Cela C, Choi BJ, Flores J, Freyberg RJ, McCabe BD, Mosharov EV, Krantz DE, Javitch JA, Sulzer D, Sames D, Rayport S, et al. (2017) Neuronal depolarization drives increased dopamine synaptic vesicle loading via VGLUT. Neuron 95:1074-1088.e1077.

Asanuma M, Miyazaki I, Ogawa N (2003) Dopamine- or L-DOPA-induced neurotoxicity: the role of dopamine quinone formation and tyrosinase in a model of Parkinson's disease. Neurotox Res 5:165-176.

Bérubé-Carrière N, Riad M, Dal Bo G, Lévesque D, Trudeau LE, Descarries L (2009) The dual dopamine-glutamate phenotype of growing mesencephalic neurons regresses in mature rat brain. J Comp Neurol 517:873-891.

Betarbet R, Sherer TB, MacKenzie G, Garcia-Osuna M, Panov AV, Greenamyre JT (2000) Chronic systemic pesticide exposure reproduces features of Parkinson's disease. Nat Neurosci 3:1301-1306.

Betarbet R, Sherer TB, Greenamyre JT (2002) Animal models of Parkinson's disease. Bioessays 24:308-318.

Blesa J, Przedborski S (2014) Parkinson's disease: animal models and dopaminergic cell vulnerability. Front Neuroanat 8:155.
Brookes PS, Yoon Y, Robotham JL, Anders MW, Sheu SS (2004) Calcium, ATP, and ROS: a mitochondrial love-hate triangle. Am J Physiol Cell Physiol 287:C817-C833.

Cannon JR, Greenamyre JT (2009) NeuN is not a reliable marker of dopamine neurons in rat substantia nigra. Neurosci Lett 464:14-17.

Cannon JR, Tapias V, Na HM, Honick AS, Drolet RE, Greenamyre JT (2009) A highly reproducible rotenone model of Parkinson's disease. Neurobiol Dis 34:279-290.

Clarkson ED, Rosa FG, Edwards-Prasad J, Weiland DA, Witta SE, Freed CR, Prasad KN (1998) Improvement of neurological deficits in 6-hydroxydopamine-lesioned rats after transplantation with allogeneic simian virus 40 large tumor antigen gene-induced immortalized dopamine cells. Proc Natl Acad Sci USA 95:1265-1270.

Costes SV, Daelemans D, Cho EH, Dobbin Z, Pavlakis G, Lockett S (2004) Automatic and quantitative measurement of protein-protein colocalization in live cells. Biophys J 86:3993-4003.

Dal Bo G, Bérubé-Carrière N, Mendez JA, Leo D, Riad M, Descarries L, Lévesque D, Trudeau LE (2008) Enhanced glutamatergic phenotype of mesencephalic dopamine neurons after neonatal 6-hydroxydopamine lesion. Neuroscience 156:59-70.

de Lau LM, Breteler MM (2006) Epidemiology of Parkinson's disease. Lancet Neurol 5:525-535.

De Miranda BR, Rocha EM, Bai Q, El Ayadi A, Hinkle D, Burton EA, Greenamyre JT (2018) Astrocyte-specific DJ-1 overexpression protects against rotenone-induced neurotoxicity in a rat model of Parkinson's disease. Neurobiol Dis 115:101-114.

De Miranda BR, Fazzari M, Rocha EM, Castro S, Greenamyre JT (2019) Sex differences in rotenone sensitivity reflect the male-to-female ratio in human Parkinson's disease incidence. Toxicol Sci 170:133-143.

Divakaruni AS, Wallace M, Buren C, Martyniuk K, Andreyev AY, Li E, Fields JA, Cordes T, Reynolds IJ, Bloodgood BL, Raymond LA, Metallo CM, Murphy AN (2017) Inhibition of the mitochondrial pyruvate carrier protects from excitotoxic neuronal death. J Cell Biol 216:1091-1105.

Drechsel DA, Liang LP, Patel M (2007) 1-Methyl-4-phenylpyridiniuminduced alterations of glutathione status in immortalized rat dopaminergic neurons. Toxicol Appl Pharmacol 220:341-348.

Drummond GB, Paterson DJ, McGrath JC (2010) ARRIVE: new guidelines for reporting animal research. Exp Physiol 95:841.

Erben L, Buonanno A (2019) Detection and quantification of multiple RNA sequences using emerging ultrasensitive fluorescent in situ hybridization techniques. Curr Protoc Neurosci 87:e63.

Feissner RF, Skalska J, Gaum WE, Sheu SS (2009) Crosstalk signaling between mitochondrial $\mathrm{Ca}^{2+}$ and ROS. Front Biosci (Landmark Ed) 14:1197-1218.

Fortin GM, Ducrot C, Giguere N, Kouwenhoven WM, Bourque MJ, Pacelli C, Varaschin RK, Brill M, Singh S, Wiseman PW, Trudeau LE (2019) Segregation of dopamine and glutamate release sites in dopamine neuron axons: regulation by striatal target cells. FASEB J 33:400-417.

Freestone PS, Chung KK, Guatteo E, Mercuri NB, Nicholson LF, Lipski J (2009) Acute action of rotenone on nigral dopaminergic neurons: involvement of reactive oxygen species and disruption of $\mathrm{Ca}^{2+}$ homeostasis. Eur J Neurosci 30:1849-1859.

Geisler S, Wise RA (2008) Functional implications of glutamatergic projections to the ventral tegmental area. Rev Neurosci 19:227-244.

Gibb WR (1992) Melanin, tyrosine hydroxylase, calbindin and substance P in the human midbrain and substantia nigra in relation to nigrostriatal projections and differential neuronal susceptibility in Parkinson's disease. Brain Res 581:283-291.

Herzog E, Takamori S, Jahn R, Brose N, Wojcik SM (2006) Synaptic and vesicular colocalization of the glutamate transporters VGLUT1 and VGLUT2 in the mouse hippocampus. J Neurochem 99:1011-1018.

Hnasko TS, Chuhma N, Zhang H, Goh GY, Sulzer D, Palmiter RD, Rayport S, Edwards RH (2010) Vesicular glutamate transport promotes dopamine storage and glutamate corelease in vivo. Neuron 65:643-656.

Ikemoto S (2010) Brain reward circuitry beyond the mesolimbic dopamine system: a neurobiological theory. Neurosci Biobehav Rev 35:129-150.

Johnson ME, Bobrovskaya L (2015) An update on the rotenone models of Parkinson's disease: their ability to reproduce the features of clinical disease and model gene-environment interactions. Neurotoxicology 46:101-116.

Jung S, Chung Y, Lee Y, Lee Y, Cho JW, Shin EJ, Kim HC, Oh YJ (2019) Buffering of cytosolic calcium plays a neuroprotective role by preserving the autophagy-lysosome pathway during $\mathrm{MPP}(+)$-induced neuronal death. Cell Death Discov 5:130. 
Kashani A, Betancur C, Giros B, Hirsch E, El Mestikawy S (2007) Altered expression of vesicular glutamate transporters VGLUT1 and VGLUT2 in Parkinson disease. Neurobiol Aging 28:568-578.

Kolber P, Krüger R (2019) Gene-environment interaction and Mendelian randomisation. Rev Neurol (Paris) 175:597-603.

Kouwenhoven WM, Fortin G, Penttinen AM, Florence C, Delignat-Lavaud B, Bourque MJ, Trimbuch T, Luppi MP, Salvail-Lacoste A, Legault P, Poulin JF, Rosenmund C, Awatramani R, Trudeau LE (2020) VGluT2 expression in dopamine neurons contributes to postlesional striatal reinnervation. J Neurosci 40:8262-8275.

Liang CL, Sinton CM, Sonsalla PK, German DC (1996) Midbrain dopaminergic neurons in the mouse that contain calbindin-D28k exhibit reduced vulnerability to MPTP-induced neurodegeneration. Neurodegeneration 5:313-318.

Martinez TN, Greenamyre JT (2012) Toxin models of mitochondrial dysfunction in Parkinson's disease. Antioxid Redox Signal 16:920-934.

Matsuda W, Furuta T, Nakamura KC, Hioki H, Fujiyama F, Arai R, Kaneko $\mathrm{T}$ (2009) Single nigrostriatal dopaminergic neurons form widely spread and highly dense axonal arborizations in the neostriatum. J Neurosci 29:444-453.

Mazzulli JR, Zunke F, Isacson O, Studer L, Krainc D (2016) $\alpha$-Synucleininduced lysosomal dysfunction occurs through disruptions in protein trafficking in human midbrain synucleinopathy models. Proc Natl Acad Sci USA 113:1931-1936.

Meiser J, Weindl D, Hiller K (2013) Complexity of dopamine metabolism. Cell Commun Signal 11:34.

Mingote S, Chuhma N, Kusnoor SV, Field B, Deutch AY, Rayport S (2015) Functional connectome analysis of dopamine neuron glutamatergic connections in forebrain regions. J Neurosci 35:16259-16271.

Mingote S, Chuhma N, Kalmbach A, Thomsen GM, Wang Y, Mihali A, Sferrazza C, Zucker-Scharff I, Siena AC, Welch MG, Lizardi-Ortiz J, Sulzer D, Moore H, Gaisler-Salomon I, Rayport S (2017) Dopamine neuron dependent behaviors mediated by glutamate cotransmission. Elife 6: e27566.

Mingote S, Amsellem A, Kempf A, Rayport S, Chuhma N (2019) Dopamineglutamate neuron projections to the nucleus accumbens medial shell and behavioral switching. Neurochem Int 129:104482.

Nussbaum RL, Ellis CE (2003) Alzheimer's disease and Parkinson's disease. N Engl J Med 348:1356-1364.

Paxinos G, Watson C (2007) The rat brain in stereotaxic coordinates, Ed 6. Amsterdam: Academic/Elsevier

Poulin JF, Zou J, Drouin-Ouellet J, Kim KY, Cicchetti F, Awatramani RB (2014) Defining midbrain dopaminergic neuron diversity by single-cell gene expression profiling. Cell Rep 9:930-943.

Poulin JF, Caronia G, Hofer C, Cui Q, Helm B, Ramakrishnan C, Chan CS, Dombeck DA, Deisseroth K, Awatramani R (2018) Mapping projections of molecularly defined dopamine neuron subtypes using intersectional genetic approaches. Nat Neurosci 21:1260-1271.

Radad K, Al-Shraim M, Al-Emam A, Wang F, Kranner B, Rausch WD, Moldzio R (2019) Rotenone: from modelling to implication in Parkinson's disease. Folia Neuropathol 57:317-326.

Ricke KM, Paß T, Kimoloi S, Fährmann K, Jüngst C, Schauss A, Baris OR, Aradjanski M, Trifunovic A, Eriksson Faelker TM, Bergami M, Wiesner RJ (2020) Mitochondrial dysfunction combined with high calcium load leads to impaired antioxidant defense underlying the selective loss of nigral dopaminergic neurons. J Neurosci 40:1975-1986.

Rocco BR, Lewis DA, Fish KN (2016) Markedly lower glutamic acid decarboxylase 67 protein levels in a subset of boutons in schizophrenia. Biol Psychiatry 79:1006-1015.

Rocha EM, De Miranda BR, Castro S, Drolet R, Hatcher NG, Yao L, Smith SM, Keeney MT, Di Maio R, Kofler J, Hastings TG, Greenamyre JT (2020) LRRK2 inhibition prevents endolysosomal deficits seen in human Parkinson's disease. Neurobiol Dis 134:104626.

Roe DF, Craviso GL, Waymire JC (2004) Nicotinic stimulation modulates tyrosine hydroxylase mRNA half-life and protein binding to the $3^{\prime}$ UTR in a manner that requires transcription. Brain Res Mol Brain Res 120:91-102.

Shen H, Marino RA, McDevitt RA, Bi GH, Chen K, Madeo G, Lee PT, Liang Y, De Biase LM, Su TP, Xi ZX, Bonci A (2018) Genetic deletion of vesicular glutamate transporter in dopamine neurons increases vulnerability to MPTP-induced neurotoxicity in mice. Proc Natl Acad Sci USA 115: E11532-E11541.
Sherer TB, Betarbet R, Testa CM, Seo BB, Richardson JR, Kim JH, Miller GW, Yagi T, Matsuno-Yagi A, Greenamyre JT (2003) Mechanism of toxicity in rotenone models of Parkinson's disease. J Neurosci 23:10756-10764.

Silm K, Yang J, Marcott PF, Asensio CS, Eriksen J, Guthrie DA, Newman AH, Ford CP, Edwards RH (2019) Synaptic vesicle recycling pathway determines neurotransmitter content and release properties. Neuron 102:786-800.e785.

Steinkellner T, Zell V, Farino ZJ, Sonders MS, Villeneuve M, Freyberg RJ, Przedborski S, Lu W, Freyberg Z, Hnasko TS (2018) Role for VGLUT2 in selective vulnerability of midbrain dopamine neurons. J Clin Invest 128:774-788.

Steinkellner T, Yoo JH, Hnasko TS (2019) Differential expression of VGLUT2 in mouse mesopontine cholinergic neurons. eNeuro 6: ENEURO.0161-19.2019.

Strand J, Stinson C, Bellinger LL, Peng Y, Kramer PR (2018) Gi protein functions in thalamic neurons to decrease orofacial nociceptive response. Brain Res 1694:63-72.

Sulzer D, Surmeier DJ (2013) Neuronal vulnerability, pathogenesis, and Parkinson's disease. Mov Disord 28:715-724.

Surmeier DJ (2018) Determinants of dopaminergic neuron loss in Parkinson's disease. FEBS J 285:3657-3668.

Surmeier DJ, Obeso JA, Halliday GM (2017) Selective neuronal vulnerability in Parkinson disease. Nat Rev Neurosci 18:101-113.

Tank AW, Ham L, Curella P (1986) Induction of tyrosine hydroxylase by cyclic AMP and glucocorticoids in a rat pheochromocytoma cell line: effect of the inducing agents alone or in combination on the enzyme levels and rate of synthesis of tyrosine hydroxylase. Mol Pharmacol 30:486496.

Tanner CM, Goldman SM, Ross GW, Grate SJ (2014) The disease intersection of susceptibility and exposure: chemical exposures and neurodegenerative disease risk. Alzheimers Dement 10:S213-S225.

Tapias V, Greenamyre JT (2014) A rapid and sensitive automated imagebased approach for in vitro and in vivo characterization of cell morphology and quantification of cell number and neurite architecture. Curr Protoc Cytom 68:12.33.1-12.33.22

Tapias V, Greenamyre JT, Watkins SC (2013) Automated imaging system for fast quantitation of neurons, cell morphology and neurite morphometry in vivo and in vitro. Neurobiol Dis 54:158-168.

Tapias V, Cannon JR, Greenamyre JT (2014) Pomegranate juice exacerbates oxidative stress and nigrostriatal degeneration in Parkinson's disease. Neurobiol Aging 35:1162-1176.

Tapias V, McCoy JL, Greenamyre JT (2019) Phenothiazine normalizes the $\mathrm{NADH} / \mathrm{NAD}(+)$ ratio, maintains mitochondrial integrity and protects the nigrostriatal dopamine system in a chronic rotenone model of Parkinson's disease. Redox Biol 24:101164

Testa CM, Sherer TB, Greenamyre JT (2005) Rotenone induces oxidative stress and dopaminergic neuron damage in organotypic substantia nigra cultures. Brain Res Mol Brain Res 134:109-118.

Wang HS, Yu G, Wang ZT, Yi SP, Su RB, Gong ZH (2016) Changes in VGLUT1 and VGLUT2 expression in rat dorsal root ganglia and spinal cord following spared nerve injury. Neurochem Int 99:9-15.

Wood-Kaczmar A, Gandhi S, Wood NW (2006) Understanding the molecular causes of Parkinson's disease. Trends Mol Med 12:521-528.

Woodlee MT, Kane JR, Chang J, Cormack LK, Schallert T (2008) Enhanced function in the good forelimb of hemi-Parkinson rats: compensatory adaptation for contralateral postural instability? Exp Neurol 211:511-517.

Yamaguchi T, Qi J, Wang HL, Zhang S, Morales M (2015) Glutamatergic and dopaminergic neurons in the mouse ventral tegmental area. Eur J Neurosci 41:760-772.

Yamaguchi T, Wang HL, Li X, Ng TH, Morales M (2011) Mesocorticolimbic glutamatergic pathway. J Neurosci 31:8476-8490.

Zhang S, Qi J, Li X, Wang HL, Britt JP, Hoffman AF, Bonci A, Lupica CR, Morales M (2015) Dopaminergic and glutamatergic microdomains in a subset of rodent mesoaccumbens axons. Nat Neurosci 18:386-392.

Zharikov A, Bai Q, De Miranda BR, Van Laar A, Greenamyre JT, Burton EA (2019) Long-term RNAi knockdown of $\alpha$-synuclein in the adult rat substantia nigra without neurodegeneration. Neurobiol Dis 125:146-153.

Zheng X, Huang Z, Zhu Y, Liu B, Chen Z, Chen T, Jia L, Li Y, Lei W (2019) Increase in glutamatergic terminals in the striatum following dopamine depletion in a rat model of Parkinson's disease. Neurochem Res 44:10791089. 